\title{
LA LEXICOGRAFÍA PLURILINGÜE INGLESA EN LOS SIGLOS XVI Y XVII: LOS DICCIONARIOS DE JAMES HOWELL ${ }^{1}$
}

\author{
Francisco Javier Sánchez Escribano \\ Universidad de Zaragoza
}

\begin{abstract}
RESUMEN
En este artículo estudiamos el desarrollo de la lexicografía inglesa de los siglos XVI y XVII y su relación con la española, francesa e italiana, especialmente con la primera. Hemos tomado los diccionarios políglotos de James Howell porque son un buen ejemplo de cómo se confeccionaban los diccionarios de entonces, a veces partiendo de diccionarios bilingües. Consideramos que su Lexicon Tetraglotton hay que tomarlo con cautela porque quizá no introduzca muchas voces nuevas; pero su Nomenclatura es un diccionario que merece la atención de los amantes de la lexicografía.

Palabras clave: James Howell; Lexicon Tetraglotton; Nomenclature.

ABSTRACT

In this article we study the development of English lexicography in the 16th and $17^{\text {th }}$ centuries and its relation with Spanish, French and Italian, especially the former. I have taken as an example James Howell's multilingual dictionaries because they are a good example of how these dictionaries were made out, sometimes taking bilingual dictionaries as the starting point. I consider that his Lexicon Tetraglotton must be taken with caution because his contribution with new words is not important; but his Nomenclature is a dictionary that deserves the attention of lexicographers.
\end{abstract}

Keywords: James Howell; Lexicon Tetraglotton; Nomenclature.

Ya son muchos los trabajos que se han hecho sobre la lexicografía bilingüe y plurilingüe del siglo XVI en adelante. Pero tanto este siglo como el siguiente, y también el XVIII, son la época dorada de la lexicografía en general y de la inglesa en particular.

Hemos elegido a este autor porque resulta ser un buen ejemplo de cómo se componían los diccionarios en esa época: partiendo de un original, se añadían voces nuevas o se copiaba directamente de otros diccionarios. Por ello resulta difícil separar en este proceso

\footnotetext{
${ }^{1}$ Ya hace años que analicé los diccionarios de James Howell (Sánchez, 1979 y 1983), pero 25 años después, y con nuevo y más material disponible, considero que mis trabajos necesitan una buena revisión y la corrección de numerosos errores propios y ajenos.
} 
los diccionarios bilingües de los plurilingües. En este trabajo trataremos del proceso y valoración de la obra lexicográfica de James Howell, cuyo propósito principal era tomar como referencia el inglés y, como veremos, apoyarse en el francés, italiano y español, según le convenía. Aprovecharemos para ver cómo va naciendo la relación lexicográfica entre el inglés y el español.

Son numerosas las alusiones que se han hecho a las aportaciones de James Howell a los estudios hispánicos y británicos, y no siempre se le ha hecho justicia. Es cierto que la diversificación de su producción escrita ha podido restar profundidad a sus obras pero, por otra parte, se ha de admitir que cubre un vacío que, si nos referimos a la lexicografía plurilingüe únicamente, abarca desde John Minsheu hasta John Stevens, éste ya dentro del siglo XVIII. El propósito de este trabajo consiste en delimitar apropiadamente la importancia de sus dos diccionarios políglotos en los que aparecen voces inglesas y españolas, presentados en un único volumen. El uso que todos los autores hicieron de la lexicografía bilingüe nos obliga a no prescindir de ella en este estudio.

En la portada principal queda expresado el contenido de este enciclopédico volumen que comprende un diccionario en cuatro lenguas, donde el inglés precede (y esta es una de las pretensiones del autor) al francés, italiano y español; la segunda parte la compone un diccionario glosado, reunido por materias, y la tercera, colecciones de refranes en las lenguas antes citadas, más otra en cámbrico, todas ellas dignas de ser consultadas por los amantes de la lexicografía. Esta portada sirve también para el primero de los diccionarios citados. Lleva la fecha de 1660. Todo él está dedicado a Carlos II, si bien cada una de las partes que lo componen va precedida de una dedicatoria diferente:

Lexicon Tretraglotton / AN / English-French-Italian-Spanish / DICTIONARY: / WHEREUNTO IS ADJOINED / A large NOMENCLATURE of the proper Terms / (in all the four) belonging to several Arts and Sciences, to Recreations, to / Professions both Liberal and Mechanick, \&c. / Divided into Fiftie two SECTIONS; / With another Volume of the Choicest / PROVERBS / In all the said Toungs, (consisting of divers compleat Tomes) and the English / translated into the other Three, to take off the reproach which useth to be cast upon Her, That / She is but barren in this point, and those PROVERBS She / hath are but flat and empty. / MOREOVER, / There are sundry familiar LETTERS and VERSES / running all in Proverbs, with a particular Tome of the British or old Cambrian / Sayed Sawes and Adages, which the Author thought fit to annex hereunto, and make / Intelligible, for their great Antiquity and Weight: / Lastly, there are five Centuries of New Sayings, which, in tract of Time, may serve / for Proverbs to Posterity. / By the Labours, and Lucubrations of JAMES HOVVEL, Esq; / Senesco, non Segnesco. / LONDON, / Printed by J. G. for Cornelius Bee, at the Kings Armes / in Little Brittaine. 1660.

Este complejo volumen se publicó sin licencia ${ }^{2}$ por un tal J. G. ${ }^{3}$, que también imprimiría el volumen de los refranes, todo ello para el conocido y rico librero Cornelius Bee (Plomer 1907: 19).

\footnotetext{
${ }^{2}$ Tampoco se encuentra inscrito en el Register of the Company of Sationers of London, lo que no es de extrañar dada la reglamentación que sobre la impresión de libros existía en esos años. En efecto, el 11 de julio de 1637 un decreto de la "Star Chamber" obligaba a que "every book had still to be entered in the Stationer's Register, and to bear the name of the printer, the author and the publisher" (Mumby 1974: 96-126). Cuando en 1641 el "Long 
Este volumen puede consultarse en la Biblioteca Nacional de Madrid donde existen dos copias $^{4}$.

\section{EL LEXICON TETRAgLOTTON}

Lo componen 394 páginas sin numerar, divididas en tres columnas, con una media de unas 30 voces en cada una, así como una sola letra guía. Son, pues, unas 90 voces por página. Se sigue el orden alfabético $A, B, C, D, E, F, G, H, I, K, L, M, N, O, P, Q, R, S, T$, $V, W, Y, Z$. La $J$ se incluye en la $I$ y la $U$ en la $V$. Anotamos la ausencia de $X$. Las voces siguen el orden: inglés (en redonda), francés (en cursiva), italiano (en redonda) y español (en cursiva). Más tarde retomaremos el alfabeto.

Uno de los propósitos manifiestos del autor es evitar todo tipo de interrupción que distraiga y desoriente al lector. Lo señala en el título que precede a la primera letra:

An English - French - Italian - Spanish / DICTIONARY. / All which four Languages do follow one another in a / regular uninterrupted ${ }^{5}$ cours throughout the / whole Work.

Ya lo había advertido en la dedicatoria al "tru Philologer", que le precede, donde se puede leer que el lector no se verá desorientado por etimologías o proverbios, en alusión clara y directa a Minsheu y a su diccionario polígloto. Otra la podemos ver en el continuo reenvío de un diccionario a otro que este autor hace, cosa que Howell quiere evitar:

The first (part) is a fower toungd Dictionary, where the English doth head the French, Italian and Spanish the three noblest languages of Europe, and they all fower come without any interruption of Etymologies, or Proverbs (which are also here in Tomes by themselves) immediately one after the other, and this will prove a great advantage to Memory, in regard of the affinity and consonance they have one with another in thousands of words, as to dispute (in English) disputare (in Italian) disputer (in French) disputar (in Spanish) ..., with multitudes more, which coming so immediately one after the other will be a great help to the retention of memory, and to carry away all the fower with more ease.

Nor will the Reder here be sent twice upon one arrand as other Dictionaries use to send him, putting him to a double labour, If you look for a badger, he wil not turn you to seek a brock, nor from a brock to a gray, nor vice versa, ..., but every word though it hath many

Parliament" abolió la "Star Chamber", el decreto quedó en letra muerta. Esto explica que sean tantos los libros que no pasaban por el registro. Esta situación duró hasta la publicación el 3 de julio de 1663 por Roger L'Estrange de sus Considerations and Proposals in order to the Regulation of the Press.

${ }^{3}$ Debe tratarse de John Grismond II. Se encontraba en la lista de impresores que en 1649 fueron obligados a no imprimir "seditious books" (Domestic State Papers, 1649-1650, pp. 522-523). En 1664 fue arrestado a instancias de la Company of Stationers por imprimir libros ilegalmente (Plomer 1907: 87).

${ }^{4}$ La copia utilizada con signatura 2/63565 lleva el sello de la BR y en manuscrito "Colleg. Paris. Soc. Jesu". La otra lleva la signatura 2/69780. Existen otras dos copias con registro procedente del antiguo catálogo que no hemos consultado. En la British Library de Londres hay una copia (71.f.4.) en perfecto estado de conservación y con un retrato del autor.

${ }^{5}$ La cursiva es suya. 
Synonymas will satisfie you by it self, and not make you tumble the leafs so often for one thing which will put one to an Impatience.

No nos da ningún dato sobre el método que siguió para coleccionar las voces pero es evidente que utilizó diccionarios publicados anteriormente y siguió su metodología, con la inclusión de voces nuevas sobre diferentes temas, hay términos que no conoce y los define, a veces es la traducción de otra lengua, otras es un término nuevo adoptado también de otra lengua, etc., porque dice en la última página de esta dedicatoria:

Let the Judicious Reder observe besides, that in this new Lexicon and Nomanclature ther be very many recent words in all the fower languages which were never inserted in Dictionary before, It is now above forty yeers since Florio, Cotgrave, and Minshew compild theirs but ther be divers words got into those languages since; Touching the English, what a number of new words have got into her of late years which will be found here; as stumming of wine, clover grasse, regalos, treatment, mobby, punch, perino, (Carriby Ilands drink) picaro, peccadillio, pantaloon, vogue, Quakers, Seekers, Levellers, Trepanners, piqueering, plundering, storming, Excise, \& $c$. and others which got in during the reign of the Long Parlement. 6

Además de lo expuesto hasta el momento encontramos una velada alusión a Percyvall y Minsheu en la última dedicatoria al lector, esta vez por parte del impresor, que lleva por título en español (está escrita en los cuatro idiomas del diccionario) "El Imprimidòr al Letor prudente". En ella se excusa de la posible existencia de numerosas faltas (son demasiadas en las tres partes del volumen) debido, dice, a la ausencia del autor (cosa más que probable $^{7}$ ) y a la falta de corregidores $^{8}$ :

En el ausencia del Autòr algunas vezes por auer la sobreintendencia de la Obra, y por falta de Corregidores harto platicos en tantos lenguajes debaxo de este Clima Insular tajado del resto del Mundo, No aurà de que espantarse si algunos yerros de la Imprimeria se topen en esta primera Edicion; Mas esperanças ay, que el Letor generoso, y juyzioso sin trompçarse a cada paja, passarà siempre más adelante hasta el sentido entero de la cosa, sin hazerse Critico, ò Curioso en demasia, porque en la lectura de libros ay una regla llena de buena moralidad, que se deve observar, Agnoscendum quod benè ignoscendum quod secùs.

Si tenemos en cuenta las alusiones a Minsheu y su reciente afirmación de que en su diccionario se encontrarán palabras nuevas y que ya hacía cuarenta años que habían sido publicados el de aquél y los de Florio y Cotgrave, podemos deducir cuáles han podido ser los autores de los que se ha servido Howell. Del primero para todos los idiomas, del segundo especialmente para el italiano y del tercero para el francés.

\footnotetext{
${ }^{6}$ Hemos comprobado que son numerosas las nuevas voces que se incluyen en los dos diccionarios y estas son solo unos pocos ejemplos.

${ }^{7}$ Hemos de recordar que ingresó en prisión el 14 de noviembre de 1642 y allí permaneció hasta la amnistía de 1650, si bien no se vio libre del todo hasta siete años más tarde, a la muerte de Cromwell. Lo amplio de su producción literaria justificaría el poco cuidado puesto en la impresión de algunas de sus obras.

${ }^{8}$ Percyvall tuvo a don Pedro de Valdés y a don Vasco de Sylva (Steiner 1970: 24) y Minsheu, como él mismo dice, tuvo "a company of certaine Strangers and Schollers at mine own charge about the Worke there" (Steiner 1970: 53).
} 
Por lo que se refiere a John Florio (1553?-1625), dedicó gran parte de su vida a la propagación de la lengua y la literatura italianas por Inglaterra. Escribió varias obras pero es más conocido por su diccionario

A / Worlde / of Wordes, / or / Most copious, and exact / Dictionarie in Italian and / English, collected by / John Florio. / Printed at London, by / Arnold Hatfield for / Edw. Blount. / 1598.

Era el primer diccionario propiamente dicho dedicado al Italiano-Inglés y uno de los más importantes del Renacimiento inglés. Durante los trece años que transcurrieron entre la primera edición y la segunda, Florio fue completándolo y cuando apareció en 1611 mereció la alabanza de sus contemporáneos, entre los que destacan John Thorius y el poeta Samuel Daniel.

En 1659, fecha en que aparece el Nomenclator de Howell, vuelve a salir una nueva edición, aumentada por el propio Florio antes de su muerte, completada y revisada por Giovanni Torriano. Esta edición debe merecer nuestra atención porque además de la notable ampliación incluye un diccionario Inglés-Italiano y 234 proverbios numerados "never before published", así como una breve introducción a la lengua italiana. Si bien Howell no pudo utilizarla (aparece un año antes de su Lexicon), es muy probable que conociera su existencia, y por ello la incluyo en el estudio comparativo.

Randle Cotgrave (murió en 1634), por su parte, parece que no publicó otra cosa que

A / Dictionarie / of the French / and / English / Tongues. / Compiled by Randle / Cotgrave. / London / Printed by Adam Islip / Anno 1611.

No obstante dedicó muchos años al diccionario porque lo había de revisar profundamente en la edición de 1623. Este volumen, según leemos en la introducción,

is a vast treasury of information about both English and French and is frequently cited by lexicographers. It has considerable interest for historians of proverbial sayings because of their frequent appearance through the work. Though preceded in time by the Dicionarie French and English (1571) (de Claude Desainliens) it is undoubtedly one of the most important interlingual dictionaries produced in the seventeenth century and was reprinted four times up to $1672 .{ }^{9}$

En la edición de 1632 Robert Sherwood añade el diccionario Inglés-Francés y el trabajo debió complacer tanto a Howell que en 1650 lo reedita totalmente revisado. Esta edición tuvo tal éxito que se hicieron hasta siete reediciones. Y aunque nuestro autor parece hacer referencia a la de 1611 cuando dice que han pasado cuarenta años desde la publicación de los diccionarios de Florio, Cotgrave y Minsheu, creo que para las voces francesas se sirvió de la de 1632 y muy probablemente de ésta de 1650 que él mismo había revisado. En el estudio comparativo utilizaremos esta edición.

En 1660 aparece una nueva edición de este diccionario mucho más ampliada y en la que se incluye una gramática, un diálogo y una serie de proverbios, todo según las últimas

\footnotetext{
${ }^{9}$ En English Linguistics 1500-1800. A Collection of Facsimile Reprints, Menston (Inglaterra), The Scholar Press
} Limited, 1968. 
normas de la Academia del Cardenal Richelieu. En ella el nombre de James Howell Esqr. se encuentra mucho más destacado. También introduce unas reglas para la pronunciación de algunas letras inglesas, otra para la conjugación de los verbos y un pequeño vocabulario por materias.

El último de los tres autores citados por Howell, John Minsheu, es sin duda el más importante y no precisamente por su originalidad como ocurría con los ya señalados. No voy a discutir su valor porque ya lo han hecho Roger J. Steiner (1970: 38-57) y Sofía Martín Gamero (1961: 83-97), ambas obras de referencia obligada, así que me limitaré a exponer cuáles fueron las obras que pudieron influir en Howell, al mismo tiempo que hacemos un pequeño recorrido por la lexicografía anglo-española.

En 1599 aparece en Londres un extenso diccionario bilingüe que Minsheu toma casi en su totalidad del de Richard Percyval, su Bibliotheca Hispanica (1591), y del de Florio (Steiner 1970: 40). Se compone de un diccionario Español-Inglés (248 págs.); de otro Inglés-Español (págs. 249 a 391), que incluiré en el estudio comparativo; de una gramática española (84 págs.), con paginación independiente, que copió de Percyvall, el Anónimo de Lovaina (1559), Stepney, Miranda y Corro, y que en parte sirvió a Howell; una colección de 64 refranes españoles y, por último, los Diálogos.

Nos interesan los dos diccionarios, pero sobre todo el Inglés-Español por ser el primero que se incluía en un diccionario bilingüe Español-Inglés. Para su composición, dice Steiner (1970: 47),

he took the glosses from the Spanish-English part of his dictionary, rearranged them in alphabetical order, set the word "vide" or its abbreviation "v", after each of them, and, in their respective position, placed the Spanish entry words of the Spanish-English part of the Dictionary.

En 1617 publica un monumental diccionario en once idiomas, si bien en las ediciones de 1625, 1626 y 1627 no aparecen ni el Británico o Galés ni el Portugués, así como tampoco el diccionario Español-Inglés. Nos referimos a The Guide into the Tongues... Resulta de sumo interés la invitación que hace en la portada a ingleses y extranjeros "if they will draw out of these one or more Languages, and place them in order of Alphabet and Table, and referre them by figures into this Booke, as they shall best like of", porque Howell la va a aceptar.

También lleva este volumen un curioso vocabulario español con la traducción inglesa y latina, y foliación independiente, en el que al lado de cada vocablo señala la página en que está y el número que tiene cada una en la Guide... A pesar de que le llevó mucho tiempo componer este diccionario, "an Ocean of trauailles, troubles, and hard sufferings, and wants, the greater part of this twenty yeeres", como dice la introducción, contó con la inestimable ayuda de extranjeros que revisaron su trabajo. Recordemos que Howell, por boca del impresor, se excusaba por las posibles faltas diciendo que no había contado con la ayuda de colaboradores.

Para el corto estudio comparativo tomaré exclusivamente las voces inglesas y su traducción al español. El número corresponde al orden seguido en el diccionario de Howell. 
Las dos primeras se refieren a la letra $A$ como primera del alfabeto y su pronunciación en inglés. Y sigue:

3. $A$, is also a prepositive article in English (and in no toung els) before words beginning with a consonant, as a Book, a Cock; but before words beginning with vowels, there is $n$, added, as an Eagle, an arbour...

$A$ serve de articulo prepositivo en Yngles (pero en ningun otro lenguaje) delante los vocablos que comiençan con consonante, mas començando con vocales se añade $n$.

Minsheu (1617): ... apud Anglos autem A præpositivum ante nomina a man.

Sherwood (Cotgrave, 1650): An; vn, une. Articles mis les Noms Substantif; C'est à dire, le premier devant ceux qui commencent par voyelle, ou par $h$ muet, comme An Eagle, un aigle. An heir, un heritier.

4. There is a proverb in French which makes much for the honor of A. viz. Il est marque à $l^{\prime}$ A, He is marked with $\mathrm{A}$, that is, He is a perfect honest man; ...

Ay un refran en Frances por la honra the A, viz. Esta señalado con A, es a saber, Es muy hombre de bien.

Cotgrave (1650): ... Il est Marqué à l'A: he is a right honest man: or, one in whom there is as much vertue, as great worth, as can be in any: From the money of France; every sort whereof hath stamped on it a particular letter, denoting the place wherein it was made; now that which is coined in Paris had on it an A, and is, commonly, of the best mettall.

5. Aaron the first Priest among the Jews; ... El primer Sacerdote entre los Judios.

Minsheu (1625): .... the High Priest of the people of Israel.

6. Abadon, or Abaddon, the spirit of strife spoken of in holy Scriptures; ... El espiritu de contienda del qual habla la sagrada Escritura.

Minsheu (1625): Abadon Abbadon... was king of the Locust, and Angell of the bottomlesse pit.

7. To Abandon, or forsake; ... Desamparar, dexar, baldonar, abaldonar.

Minsheu (1617 y 1625): des-Amaparár (à des. i. deshazér al amparo, o dexár de Amaparár, i altogether to leaue to parár protect, (\& Amparár ab am i. circum, circa, about, $\&$, i. sistere).

8. To Abandon one's self to pleasures, or play, \&c; ... Yr tras sus antojos, andar tras el juego.

Minsheu (1625): to Abandon himselfe, or giue himself wholly ouer to his pleasures... yr tras sus antojos.

9. Abandoned or forsaken; ... Desamparado, dexado, baldonado.

Minsheu (1599): vide Abandonado.

Minsheu (1625): des-Amparádo.

10. Abandoned or forsaken by the Physitians; ... Desauciado. 
11. An abandoning or quite forsaking; ... Desamparado, desamparamiento, baldonamiento.

Minsheu (1599): vide Abandonamiénto.

Minsheu (1617 y 1625): des-Amparo.

12. The abase, or bring down; ... Abaxar, deprimir, amilanar, someter, humiliar.

Minsheu (1599): To abase or bring low, vide Abatir, Abaxar.

Minsheu (1617 y 1625): To abase or bring low, Abaxar.

A partir de estas diez voces, las primeras del Lexicon de Howell, podemos deducir que en éste las voces inglesas son las verdaderamente importantes (no olvidemos que éste es su propósito, que confirma en repetidas ocasiones) y que las españolas dependen de las anteriores, incluidas las francesas e italianas, que debe a Florio. Por regla general nuestro autor da más de un equivalente español para la correspondiente voz inglesa, aunque a la hora de dilucidar su valor lexicográfico deberemos ser muy prudentes. En efecto, incluye casi todo el diccionario español de Minsheu (edición de 1617), añadiendo palabras de significado similar. Sin embargo, "his work must therefore be used with caution, as the older lexicographer treats his words with greater precision, and from the first sources", dice Leo Wiener (1899: 9) acertadamente.

Amado Alonso (1951: 326), por su parte, no deja claro en "Reseñas" a cuál de los dos diccionarios se refiere cuando dice de Howell que "tenía más atrevimiento que conocimiento del español, y lo que no haya tomado de otros libros (Minsheu en primer lugar, que ya era poco de fiar) no es materia de confianza". Supongo que se refiere al Lexicon... y, como puede verse, coincido con su apreciación en este caso. En numerosas ocasiones demuestra su dependencia del italiano y el francés, idiomas que dominaba mejor que el castellano.

Por lo que se refiere al inglés, Howell advierte en la introducción "To the tru Philologer", ya citada anteriormente, que se añaden nuevos términos entre los que se encuentran algunos hispanismos. Ocurre en los cuatro idiomas. Pero vayamos por partes.

Por lo que se refiere al alfabeto, casi todas las letras las describe ${ }^{10}$, algunas de una manera curiosa. Tomaremos solamente las que tienen alguna significación para el castellano:

B, The First consonant, and second Letter of the Alphabet, is in Italian and Spanish used promiscuously in some words for $V$, as Nerbo a sinew instead of Nervo; Serbo a servant instead of servo.

D, Is so dainty of letter, that she admits of no other consonant next her but R: In Spanish she thawes as it were, very often into th, as Dados dyce is pronounced dathos: Dedo a finger, detho: with divers other words.

$\mathrm{K}$, The Italian and Spaniard know not K, but $\mathrm{C}$ makes it superfluous.

\footnotetext{
${ }^{10}$ También lo hace en su Gramática Inglesa (1660) con una exhaustiva descripción de todas las letras, haciendo hincapié en su pronunciación y, en su caso, comparándolas con las de otros idiomas.
} 
$\mathrm{N}$, The third of the fower liquids, in Spanish it hath very often a title above, as $\tilde{n}$, and then t'is pronounced as if $I$ came immediately after, as Año a year, is pronounced Anio; Baño a bath, Banio \& c.

$\mathrm{V}^{11}$, may be called an Amphibolous, or mongrel letter, for she may be sayed to be as much Consonant as Vowell, in all the foure languages for the most part.

$\mathrm{W}^{12}$, The English toung hath $\mathrm{W}$ in her Alphabet more then French, Spanish, or Italian, who aknowledge no such letter.

En cuanto a la organización del diccionario, el problema que se plantea en el ordenamiento de las entradas lo soluciona siguiendo la tradición de ordenamiento etimológico de los lexicógrafos del siglo XVI, aunque a veces se complique un poco cometiendo los mismos errores que ellos. El uso de artículos o preposiciones es muy común. Pondremos un ejemplo con las entradas en los cuatro idiomas:

Equippage; Equippage; Apparecchio, provigione; Aparejo, pertrecho, atuendo, apercibimiento, recaudo.

In full Equipage; Ordonné, arroyé; Apparechiato, proveduto; Aparejado, pertrechado, apercebido.

The Equipage of a Princes Court, or Camp; Bernage, barnage; Apparecchi di un Principe; Pertrecho de Principe.

To put in Equipage; Arroyer, equipper; Ordinare, fornire, guernire; Adereçar, aparejar, enjaezar.

To Equipp, or store with furniture; Equipper, equipper, ordonner; Provedere, fornire; Pertrechar, provéer.

Equipped, or made ready; Equippé, equipé, ordonné; Apparecchiato, fornito; Pertrechado, proveydo.

Otra de las características es el uso de definiciones en los cuatro idiomas, tanto si conoce el término como si no lo hace:

Alimony, or maintenance which the husband by law ought to allow his wife; Aliment, ou l'appenage que le mary est oblige de donner a sa femme; L'intrata ch'il marito ò obligato di dar' alla sua moglie; Lo que el marido està obligado de dar a su muger por sustentarse.

Avoir de pois, a kind of weight; Un Espece de poids, dont seize onces font le libre, \& c. 112 livres la centaine; Una sorre di peso dove 16 oncie vanno per una libra; Il peso de haure en el qual, la libra pide 16 onças.

The Bumkin in a Ship; Chicambault, une piece de bois long or gros attaché de un baut (bout), or par le dedans du navire, avec des amarres au masterel, or servant d'omurer la

\footnotetext{
${ }^{11}$ En ella se incluye una serie de palabras que comienzan con $V$, como Vacancy...Vacacion, seguida de otra que lo hace con $U$, como An Ulcer... Ulcera, deviesso, siguiendo otras con $V$ o con $U$.

${ }^{12}$ Utiliza tanto $W$ como $V V$.
} 
misaine, or le beaupre, quand le navire va a course, où a la bouline; Sorte de antenna da nave; Especie de antenna en navio.

A Borracho, or Lether Bottle which they use much in Spain; Bourache, bourraquen, bourrabaquin; Sachetto, fiasco de cuoio; Borracha. ${ }^{13}$

A Chancell; Le plus saint endroit de l'eglise; La parte piu sacra della Chiesa; Il luogo piu santo de la Yglesia. ${ }^{14}$

Cognisance, or Crest of Armes; Cognissance, la creste des armoiries, des nobles que leurs serviteurs souloyent jadis porter sur les manches de leurs livréts, timbre; Cresta de arme; Cresta de armas.

The Flap covering the veesel of the Throat; La languette qui couvre le col des poulmons, la loüette de la gorge; La languetta che copre il polmone perche non vi vada il cibo; Gulilla.

A Maior, or Mayor of a City or Town; Le Maire, provost, gouverneur d'une ville; Il maggiore, sindico, ò magistrate principal del luogo; Corregidor, Mayor.

En su favor podemos decir que su obra es una simplificación de la Guide de Minsheu y que su importancia radica en hacerla asequible a un mayor número de público. Su dependencia de aquel autor ya ha sido discutida. No obstante, considero que esta obra de Howell debe ser incluida en todo estudio referente a la tradición lexicográfica inglesa en su relación con otros idiomas, que puede resumirse en pocas palabras.

Es en la segunda mitad del siglo XVI cuando aparecen algunos diccionarios bilingües, preferentemente incluidos en Gramáticas. Tal es el caso del pequeño vocabulario, pomposamente llamado "diccionario", que se encuentra en la traducción de las Reglas Gramaticales (salió en 1560) de Antonio de Corro que hizo John Thorius (aquél que alabaría el diccionario de John Florio en 1611):

The Spanish / Grammer: / With certaine Rules teaching both the / Spanish and French tongues. / ... / Made in Spanish, by M. An- / thonie de Corro. / With a Dictionaries adioyned vnto it,... / By Iohn Thorius. Graduate in Oxenford. / Imprinted at London by Iohn Wolfe. / 1590.

El diccionario consta de 14 páginas y no de 7 como dicen S. Martín Gamero (1961: 80) y Amado Alonso (1951: 326).

\footnotetext{
${ }^{13}$ Comparar con "A Bottle; Bouteille; Fiasco, zucca botaccio; Flasco, bota, borracha".

${ }^{14}$ Le domina el subconsciente. Toma la forma italiana a la que tiene cierta tendencia y que es el idioma que mejor domina. Basta leerse los dos diccionarios.
} 
En 1591 se publica en Londres la Bibliotheca Hispanica de Richard Percyvall ${ }^{15}$, que sería el primer diccionario Español-Inglés propiamente dicho. La Gramática consta de 34 páginas y el Diccionario de 184. Es poco probable que Howell utilizara este diccionario, pero he de señalar su importancia no sólo por ser el primer diccionario Español-Inglés, sino también por ser la fuente principal de Minsheu. Está recopilado a partir de los de Nebrija, Las Casas y Corro, y con la nada despreciable ayuda de dos prisioneros españoles, don Pedro de Valdés y don Vasco de Sylva.

Ese mismo año aparece en Londres un pequeño manual, The Spanish School-Master, de William Stepney, con siete Diálogos, Refranes y un Vocabulario. Los primeros son bilingües y todos menos el séptimo están sacados del Vocabulare de Berlaimont. Los refranes son 102 y no he podido precisar cuál haya podido ser su fuente. Por lo que respecta al Vocabulario, se trata de un diccionario de palabras agrupadas por materias (del cielo, de los cuatro elementos, etc.) sin que sigan ningún orden alfabético.

No fue en Inglaterra donde aparecieron los vocabularios políglotos sino en los Países Bajos, y más concretamente en la cosmopolita e industriosa ciudad de Amberes, como ya hemos apuntado con Steiner. En 1530 lo hace la primera edición del Vocabulare de Noel de Berlaimont o Barlement, de la que no se conserva ningún ejemplar. Sí existen algunos de la segunda, de 1536. En estas primeras ediciones era un texto bilingüe. Sin embargo, a partir de la de 1551 comienza su desarrollo en ediciones políglotas: francés, flamenco, latín y español.

En 1576 el impresor Henry Heyndrick añade el inglés y el alemán, con lo que este volumen alcanza ya las seis lenguas:

Colloqves ov Dialogves / avec un dictionarie / en six langues: Flamen, Anglois, Alleman, / François, Espainol, \& Italien / ... / A Anvers, / Chez Henry Heyndrick, au Cemitierre nostre Dame, / á la Fleur de Liz. 1576.

Las voces están agrupadas según sus iniciales en flamenco, pero sin guardar un orden alfabético. El inglés se encuentra destacado con tipografía gótica. Al final hay unas cortísimas reglas para la pronunciación del español, así como la enumeración de los artículos y pronombres.

\footnotetext{
15 Según Steiner (1970: 22, nota 4) "Many polyglot or bilingual works from Belgium were translated, registered, and published in England in the two decades or so before Percyval compiled his dictionary. Most of the following examples include Spanish as one of the languages:

1568 Thresor de tous les livres d'Amadis de Gaule (Antwerp, 1560), trans. Thomas Paynel, The Treasurie of Amadis of Fraunce (London, 1568).

1578 Dictionaire colloques ou dialogues en quattre langues fflamen, ffrancoys. Espaignol. Et italien, withe the Englishe to be added thereto. Licensed September 12, 1578.

1580 Synonymorum sylva of Simon Pelegromius (first ed. 1537) Trans. "H. F." converted the Flemish headings or topics into English (London, 1580).

1585 Nomenclator omnium rerum propia nomina variis of Adrian Junius was published at Antwerp, 1557 et seqq. Trans. (i.e. the addition of English) John Higgins (London, 1585)".
} 
Otra edición, de 1584, se diferencia de la anterior en que sustituye el alemán por el latín. La portada viene presentada en latín, español y francés. Curiosamente el latín se encuentra en primer lugar pero la agrupación según las iniciales se hace según el flamenco, lo que me hace pensar que estaba dedicado a los hablantes de ese idioma. El inglés también se encuentra destacado en tipografía gótica.

A pesar de la popularidad que alcanzaron en Inglaterra estos Vocabularios desde su aparición hay que esperar más de un siglo para que se publicase allí la primera edición. La lleva a cabo el anónimo E. G. para Michael Sparke. El volumen se presenta ya en ocho lenguas:

New / Dialogves or Colloquies, / and, / A little Dictionary of Eight Languages / Latin, French, Low-Dvtch, High-Dvtch, Spanishe, Italien, English, Portugvall. / A Book very necessary for all those that Studie / these Tongues, either at home or abroad. / Now perfected and made fit for Travellers, young / Merchant and Sea-Men, especially those that desire to attaine the use of these Tongues. / London / Printed by E. G. for Michael Sparke junior, and are to be sold niere / the Exchange and in Popeshead Palace. 1639.

El Dr. R. Verdeyen (1926, 1925 y 1935) ha escrito sendos artículos sobre las diferentes ediciones de los Vocabulare, coloquios y diccionarios políglotos, y Caroline B. Boulard (1933) completa el trabajo de aquél con una nueva relación de otros diccionarios políglotos que tanto abundaron (ambos autores son un referente obligado para este trabajo). También encontraremos algunos en Steiner (1970: 19).

Como puede deducirse, Howell tenía donde elegir. Es de suponer que conocía la existencia de estos diccionarios y, probablemente, que utilizó alguno de ellos para su aprendizaje de idiomas. Sin embargo, me inclino a pensar que es a los tres autores que cita y sobre todo a los diccionarios políglotos de Minsheu, a los que debe la casi totalidad de su Lexicon Tetraglotton. Este diccionario tiene el mérito de llenar el vacío de la segunda mitad del siglo XVII y, sobre todo, de apoyar el interés por los idiomas que los proyectos de matrimonios reales habían despertado en Inglaterra. No aporta innovaciones de relieve, pero es el único diccionario de esta época que incluye nuestro idioma. Otra de sus características, como ya he apuntado, es que con él el autor simplifica la monumental obra de Minsheu y la hace asequible a un público menos culto. Por ello extraña que Roger J. Steiner ni tan siquiera lo nombre, si bien resulta comprensible si este autor busca las fuentes principales, como ya hemos citado, y su estudio se refiere solamente a diccionarios bilingües español-inglés.

\section{EL VOCABUlario PARTICULAR O NOMENCLATURA}

La segunda parte de este monumental volumen, con fecha 1659, lleva por título

A Particular / VOCABULARY / OR / NOMENCLATURE / In English, Italian, French, and Spanish. / Of the proper Terms belonging to several Arts and Sci- / ences, to Recreations, to common Professions and Callings both Libe- / ral and Mechanick, \& c. /VOCABULARIO PARTICOLARE, ô / NOMENCLATURA / Italiana, Francese, Spagnuola, Inglese, / Delli Termini proprii, à qyualunque Arte \& Scienza, alle / Recreationi, \& Professioni cosi Liberali, come Mechaniche, \& c. / VOCABULAIRE PARTICULIER, 
ou / NOMENCLATVRE, / Françoise, Italienne, Angloise, Espagnole, / Des Termes propres aux Arts, \& Sciences, aux Re-/ creations, aux Vocations Liberales et Mechaniques, \& c./ VOCABVLARIO PARTICULAR, ô / NOMENCLATURA / Española, Inglese ${ }^{16}$, Italiana, Francese, / De los Terminos propios a las Artes y Ciencias, a las Recreationes, y Vo- / cationes Liberales y Mechanicas, \&c. /Labore \& Lucubrationibus JACOBI HOWELL Arm; / Maridunensis. / Inter Eruditos Cathedram habeat Polyglottes. / LONDON / Printed by Thomas Leach, ${ }^{17} 1659$.

Howell lo dedica

To the Pair-Royal of PEERS, / WILLIAM, / Lord Marquis of Hartford, \& c. / THOMAS, / Earl of Southampton, \& c. / IOHN / Earl of Clare, \& c.

a los que llama "Lords of these Languages", refiriéndose a las cuatro en que está compuesto.

Según afirma su autor en la introducción, “To the knowing Reader”, esta Nomenclatura le ha costado mucho trabajo, si bien confiesa haber contado con alguna ayuda, sobre todo de Monsieur de Novilliers:

It had required extraordinary Labor to have done it in one Language only, more in two, much more in three but to perform it in fower (which was never done before) let the discreeter sort of Readers judge how it would puzzle one's pericranium; I confesse to have had some single helps in divers things that did facilitat the Thing, and Monsieur de Novilliers did facilitat the Thing, and Monsieur de Novilliers did contribute more than any.

Howell advierte al lector de que no debe tomar esta obra como una curiosidad sino que ha de ser muy útil para escritores, poetas, oradores, letrados y seglares. Seguidamente da cuenta de algunos de los términos más difíciles que se podrán encontrar, algunos de ellos de origen español, como ya hiciera en el Lexicon. El último ejemplo que da es el siguiente: "...you shall not find the Egg, but you will have the Yelk, the White, and the Shell together in all the fower languages, which much differ in them all, as in Italian 'tis, Il torlo, la chiara, la guscia; In French, Le moyeu, l'aubin, la coque; In Spanish, Yema, clara, cascara".

El texto se encuentra distribuido en dos columnas por página, y las voces y glosas siguen el orden: inglés (redonda), italiano (cursiva), francés (redonda) y español (cursiva). Es éste un diccionario compuesto por 53 Secciones, cuyos términos no guardan orden alfabético alguno. Mucho me temo que casi nadie se ha dado cuenta del número de Secciones. El propio autor, en la introducción antes mencionada, dice que son 50 (lo mismo dice Martín Gamero (1961: 122); Amado Alonso (1951: 326) dice 52). En el índice salen 52, pero una lectura detenida del mismo nos confirma que el editor ha incluido dos en la 32: Fortification, with its terms y Perfumes, odors, and sweet smells, lo que nos hace un total de 53. Este buen editor nos complica más el asunto porque en el interior también figuran al

\footnotetext{
${ }^{16}$ Otra vez la influencia italiana.

${ }^{17}$ Este impresor también tuvo problemas con la ley y en 1662 "he was arrested at the instance of Sir John Birkenhead for printed seditious literature "with a base stollen edition of por Hudibras" [Domestic State Papers, Charles II, vol.49 (19)]. Debió tener una sola imprenta y un ayudante (Plomer 1907: 114).
} 
final 52. Pero es que en el interior nos encontramos con esta numeración: 31, Fencing, and the terms thereunto belonging; 33, Fortifications; 32, Perfumes, Odors, or Sweet Smells; y de nuevo 33, por lo que repite este número, Habits, or apparel for men. Hemos de añadir que algunos títulos del índice tienen variaciones con respecto a los que encabezan las secciones. En efecto, el editor hace bien en quejarse de la falta de "corregidores" como ya hemos apuntado.

Daré los títulos de las diez primeras según aparecen en el índice:

Tabla de los Capítulos comprehendidos en esta NOMENCLATURA:

Sect. I. An Anatomy of the outward, and inward parts of humane body; Una Notomia delle parti interiori ed esteriori del Corpo umano; Une Anatomie de parties interieurs, \& exterieures du corps humain; Una Anatomia de las partes internas y externas del cuerpo humano.

Sect. II. Of horse, and horsemanship, with the peculiar termes and apurtenances thereof; I cavalli, \& la cavallerizza con i sui termini; Les chevaux, \& l'ecurie avec leurs termes; Los cavallos, y la cavalleriza con sus terminos.

Sect. III. Of Hunting, or Venery, with their proper terms, and of the wild beasts and dogs that serve for that sport; Le fiere, \& la lor caccia, con $i$ termini propij di quella; La veneria, ou la chasse avec les propres termes d'icelle; Las fieras y su caça, o la montaria con sus terminos.

Sect. IV. Of Fawconry, or Hawking and all birds of prey; La struzzeria, ó falconeria, \& gli vcelli di rapina; Les oyseaux de proye, \& la Fauconerie; Las Aves di rapiña, y la Falconeria.

Sect. IV. War and Souldiery, with the military terms thereof; La guerra, \& I suoi termini; La guerre, \& ses termes; La guerra, y sus terminos.

Sect. VI. Sea-faring affairs, and navigation, with their multitude of terms; $L a$ Navigatione, \& termini marineschi; La Navigation, \& termes de la marine; La Navegacion, y terminos marineschos.

Sect. VII. Orders of Knight-hood throughout Christendom, either Regular or Secular; Ordini di Cavaglieri per tutta la Christianita, Regolari, \& Secolari; Ordres de Chevaliers par toute la Christiente Reguliers, y Seculiers; Ordines de Cavalleros por toda la Christianidad, Reglares y Seglares.

Sect. VIII. Religious Orders, and cloistered reclused Monks, Fryers, and others, according to their Antiquity and names \& c.; Ordini Religiosi, come Manachi, fratri \& altri secondo la lor antichita \& appelationi; Ordres Religieux selon leurs antiquitez, \& c. Ordines Religioso segun su antiguedad.

Sect. IX. Ecclesiastical Dignities, and Titles in the Roman Church; Ordini ecclesiastioi second il lor grado; Dignitez ecclesiastiques selon leur degrez; Dignidades ecclesiasticas conforme a sus grados.

Sect. X. The differing Sects, and Opinions in Christian Religion, with the ages, and times of the Sectaries; Opinioni differenti nella Christiana Religione, col nome, \& secolo delli sotarij; Differentes opinions en la Religion Chrestienne avec les noms, \& le temps des 
sectaires: Los differentes pareceres en la Religion Christiana con los nombres, y el tiempo de los Herejes.

Las 43 restantes se refieren a términos de arquitectura, jardinería, bibliotecas, hierbas, animales domésticos, enfermedades, fortificaciones, etc.

Considero exagerada, imprecisa y poco clara la afirmación de Amado Alonso (1951: 326, ya citada anteriormente) acerca de la insignificante aportación de Howell a la lexicografía, sobre todo porque el lingüista español parece desconocer la composición de este complicado volumen y los dos diccionarios diferentes que en él aparecen. Comparto, en parte, su opinión sobre el Lexicon pero no sobre la Nomenclatura:

... el del inglés James Howell, A particular Vocabulary or Nomenclature in English, Italian, French and Spanish of the proper terms belonging to several arts and sciences, London, 1659 ( $2^{\mathrm{a}}$ ed. 1660, con el título de Lexicon Tetragloton y algún cambio en la agrupación de los materiales), que ordena las palabras por materias en 52 secciones (caza, navegación, vinos y' bebidas, partes del cuerpo, etc.), lo cual suena muy prometedor y aún más su advertencia al lector de que encontrará en su Lexicon muchísimas palabras recientes en las cuatro lenguas nunca antes insertas en Diccionario; pero Howell tenía más atrevimiento que conocimiento del español, y lo que no haya tomado de otros libros (Minsheu en primer lugar que ya era poco de fiar) no es materia de confianza.

Leo Wiener (1899: 9) afirma lo mismo del Lexicon, pero refiriéndose a la Nomenclatura dice que "is of great and original value. It is a mine of rare expressions in every imaginable field of human activity, and is the result of an extraordinary industry". Y continúa:

What a pity we are not informed of his Spanish source. The number of words contained in these sections that are not to be found in the dictionary of the Academy or in Tolhausen is appalingly large, and many puzzling passages in the old Spanish writers are easily explained by means of this part of Howell's work.

Efectivamente, se trata de un trabajo muy original sobre todo por estar escrito en cuatro idiomas y por ser el inglés el que encabeza las secciones, al igual que ocurría en el Lexicon. Por lo que se refiere a sus aportaciones a la lexicografía española, de las que desconocemos su fuente, habrá que utilizarlo con mucha prudencia porque, como ya lo señalaba Amado Alonso, Howell tenía más atrevimiento que conocimiento del español. Ya mientras extraía las versiones inglesa y española de las Secciones podía comprobar su dependencia del italiano, idioma que sí dominaba. Sirvan de ejemplo estas tres:

Sección VI: ... La Navigatione, \& terminio marineschi ... La Navegacion, y terminos marineschos.

Sección VII: ... Ordini de Cavaglieri per tutta la Christianita, Regolari ... Ordinis de Cavalleros por toda la Christianidad, Reglares..

Sección XLVIII: ... Termini Chymici, \& gli piu scuri illustrati ... Terminos Chymicos, y los mas escuros ilustrados.

Tiene razón Howell cuando basa la originalidad de su trabajo en que nunca se había publicado ninguno parecido si se refiere a Inglaterra, si bien, como ya hemos visto con anterioridad, antes de que Percyvall publicara su obra ya se habían traducido al inglés diferentes diccionarios. Este tipo de diccionarios políglotos ya se venían publicando en los 
siglos XVI y XVII junto con los Vocabulare ${ }^{18}$. De todos ellos nos interesa solamente el de Adriano Junio (Adriaen de Jongh) por ser el único en el que figuran juntos el inglés y el español en alguna edición. La primera de ellas apareció en Augsburg en 1555 (nosotros hemos utilizado la de 1567 de Leyden) y contiene voces en latín, griego, alemán, flamenco, francés, italiano y español, con la siguiente portada:

Nomenclator, / Omnium rervm / propia nomina / variis lingvis / explicata / indicans: / Hadriano Ivnio medico avctore. / Antverpiae, / Ex officina Christophori Plantini / M.D.LXVII. / Cum privilegio.

En las primeras páginas de este Nomenclator Adriano Junio da una lista de los autores de que se ha servido para compilar tan erudita obra. En su índice aparecen 85 capítulos o secciones (S. M. Gamero dice 89) sin numerar. Se trata de un Nomenclator mucho más completo que el de Howell y ya contenía numerosas voces en inglés y español, así que no habrá que esperar a la edición de 1606 como dice la autora española. He tomado al azar las páginas 81 y 82 y de ellas he entresacado las siguientes voces:

Sepia (en griego, alemán y flamenco), G. Seche. IT. Sop. H. Siba. ANG. à cuttle, cuttel.

Squatina (...), G. Ange ou angelot de mer. IT. Squadra, squaia Venetis, angelo Liguribus. H. Lixa. ANG. Skate.

Testudo (...), G. Tortue, tortugne. IT. Galana, tortuga. H. Galapago. ANG. Schelcrab.

Thynnus (...), G. Ton, tonnine. IT. Ton, tonnina, tono. H. Atun, ANG. Tuny.

Tinca Auso, merulam lacustre putat esse Scaliger (...), G. Tache. IT. \& H. Tinco ó tenca. ANG. Tenche.

Turbo, concha Virg. (...). H. Caramuyo, caracol, almeja. ANG. á Perposs.

Xiphias, gladius Plinio (...), G. Emperador. IT. Pesce spada. ANG. Swordefysshe.

Vermis (...), G. Ver. IT. Verme, vermino. H. Gusano. ANG Worme.

Apis (...), G. Mouche à miel. IT. Ape. H. Abeja. ANG. á Bye.

De ellas en la Nomenclatura de Howell sólo aparecen la segunda y la cuarta:

Scate, ray, or thornback; Una razza, arzila in Roma; une raye; una raya.

Tunny fish; Un tonno; Un ton; Un atun.

Bueno será que pongamos unos cuantos ejemplos de los términos que Howell incluye en este curioso y, a mi juicio, excelente ejemplo de la lexicografía inglesa plurilingüe del siglo XVII. Como ya hiciera en el Lexicon, también aquí, a falta de términos exactos, utiliza definiciones:

\footnotetext{
${ }^{18}$ Como ejemplo donde muy temprano figuran el inglés y el español juntos citaremos a "Le dictionaire des huict Langages: c'est a sçavoir Grec, Latin, Flamen, François, Espagnol, Italien, Anglois, \& Aleman: fort utile \& necessaire pour tous studieux \& amateurs des lettres, nouvellement imprimé à Paris, corrige \& revue, Avec privilege (Paris, Pasquier le Tellier, dessus la porte St. Marcel, 1548). A close revision of other editions (Antwerp, 1534; Venice 1537-41)" (Steiner 1970: 19, nota 1).
} 
De la 1, que es muy larga (11 páginas), An anatomy of the Inward and Outward parts of Human Body, podemos citar:

The Brain or seat of the Animal Spirits; Il ceruello; Le cerveau, la cervelle; El celebro, los sesos, el meollo de la cabeza.

The Arteries or ligaments, whence is the pulse and movement of the vital spirits; Le Arterie, cioè, il moto de gli spiriti vitali, onde le più conosciute sono nominate polsi nelle gionture delle braccia, con le mani: Les arteres, c'est le mouvemnet des esprits vitaux, don't les plus cognus sont appelez pouls es jointures des bras, \& des mains: Las Arterias, el moto $^{19}$ de los espíritus vitales de las quales las mas conocidas se llaman pulsos en las muñecas y otras partes.

To cast amorous glances; Lampeggiare con gl' occhi, come sogliono fare le donne innamorate; Esclairer, esclater, où carrillonner les yeaux, comme ont accoustumé de faire les femmes amouseuses; Repicar con los ojos, como suelen hazer las mugeres enamoradas.

To half hoodwink ones self, as the Venetian Dames use to do; Guardare à squarciasacco cioé, l'huomo ascondendosi la faccia co l' ferrainolo, la donna co' l' velo, \& apredolo un poco, come fanno (massimamente in Venetia) le donzelle \& alter che vogliono parere d' esser an ch' esse donzelle; Regarder par le trou, c'est à scavoir, l'homme se càchant la face avec le manteau \& la famme avec son voile, l'entr' ouvrant un peu, comme font (mesmement a venise) les filles, \& d'autres aussi qui veulent sembler d'estre filles; Mirar con capotillo, mirar lasciuamente \& c.

De la 2 (9 páginas), Of Horses and horsemanship, with peculiar Terms, and Appurtenaces thereof, la primera entrada es

A Spanish or Cordovan Ginet; Un gianetto di Spagna, cavallo di Còrdova ò d'Andalosìa; Un genet d'Espagne, cheval de Cordovë, où de Andalousie; Cavallo, ginète.

Sigue una excelente descripción tanto de diferentes tipos de caballos como de sus partes y aderezos.

La 6, Of seafaring Affaires, and Navigation with their peculiar termes, contiene numerosos términos españoles y no pocas definiciones, algunas muy largas. Hay dos entradas seguidas para "estrecho de mar" donde el autor confunde "golfo" y "estrecho":

A Golf, bosome, or arm of a sea twixt two capes; Un golfo, seno ó braccio ddi mare; Un goulfe sein, ou bras de mer; Un estrecho de mar.

A streight, or narrrow sea; Uno stretto di mare; Un destroit de mer; Un estrecho de mar. $\& c$.

The last oars; L' ultimo remo della Galera; La cerniere rame de la galere; El tercerol,

Ropes that tie the sayl-yard; Corde da atraccar le vele all'antènne; Cordes à attacher les voiles al'antene; Amantillos, sogas con que se atan las velas a las antènas.

\footnotetext{
${ }^{19}$ Otra palabra italiana.
} 
The scuttle rope; La corda con che s'alza la vela della gabbia; La corde auec laquelle on monte le bourset de hune, la voile de la hune; El chúfaloète, soga con que se alça el juanete.

The scuttle y otras partes o elementos de los barcos vuelve a repetirlo en otra entrada dando para los otros tres idiomas definiciones como "Otros terminos marinescos, \&c" $o$ "Las cosas pertenecientes à todos los mastiles de un navio".

A hot African wind; Vent de galerne; Vento Affrico; Gallego viento. En esta entrada cambia el orden de los idiomas francés y luego italiano.

La 7, dedicada a las Ordines de Cavalleros por toda la Cristiandad, ò Regulares, aprovados del santo padre, ò Seglares instituidos por Principes Temporales, debe merecer la atención del lector por su interés.

Otros términos españoles que aparecen en otras secciones, algunos de los cuales, dada su traducción, supongo que desconocía, pueden ser: borraja (también la llama lengua de buey), garvanços y havas frescas nuevas (green peale y pease), calauriada (white wine and red mingled), fungos, aloja, azícares (a morisco spur that hath but one poiet), rabadán (the chief shepherd), brucho, garnacha (An Aldermans gown), chinelas, majuelo (a new vine), Vbas de almunecar (A long sort of grapes), y tantos otros. Insisto, considero que es un excelente diccionario para ser tratado por cualquier lexicógrafo.

Podemos continuar con los precedentes de la lexicografía plurilingüe. En 1606 se publica en París una edición donde el inglés y el español figuran juntos en un mayor número de voces. Se publicaron numerosas ediciones similares a ésta. Destacaré una de 1619 sin lugar de publicación (S. Martín-Gamero sugiere Ginebra):

Nomenclator / octilingvis, / Omnium Rervm / propria nomina continens. / Ab adriano Ivnio antehac collectus. / Nunc veró renouatus, auctus \& in capita LXXVII / sic distinctus, capita ipsa ad eundem modum se / consequuntur. / Accessit huic postremae editioni alter Nomenclator / é duobus veteribus Glossarüs. / Hermanni Germbergii / Opera \& studio. / Cum Indice rerum \& studio. / Cum Indice rerum \& Capitum. / Ex typographia / lacobi STOER. / M.DCXIX.

Este Nomenclator consta de 77 capítulos y contiene un diccionario agrupado por materias en latín y griego, así como unos glosarios en las mismas lenguas. Por lo que se refiere al primero es similar al de 1567 excepto en el número de capítulos.

Sofía Martín-Gamero, por su parte, afirma que "esta obra se hizo muy popular, y en ella se inspiró Minsheu para su Guide into the Tongues; Howell, para su Lexicon, y Bathe, para su Jапиа" (1961: 69-70), y más tarde cita a Amado Alonso diciendo que Howell copió a varios autores anteriores, especialmente a Minsheu (1961: 122-123), con lo que la imprecisión de la que acusaba al último queda aumentada con estas palabras de la primera. Creo que el Nomenclator de Adriano Junio sirvió a Howell de modelo e incluso de alguna información, pero nunca podrá decirse que el del autor galés es un plagio del de Minsheu. Sugiero que a las fuentes les apliquemos el calificativo de desconocidas. Estamos, como decía Leo Wiener, ante una obra importante. 
Creo, por otra parte, que la obra de Howell nada tuvo que ver con el Janua Linguarum de dos jesuitas irlandeses afincados en Salamanca. He consultado las tres principales ediciones: la de 1611, en latín y español; la de 1615, traducida por el inglés William Welde, que sustituye el español por el inglés, y la de 1617, de Jean Barbier, publicada en Londres en cuatro idiomas:

Ianva / Lingvarum / Qvadralingvis. / Or / a Messe of Tongves: / Latine, English, French and Spanish. / Neatly Serued vp together, for a wholesome / repast, to the worthy curiositie of / the studious, / Londoni, Excudebat R. F. impensis Matthaei Lownes, / M.DC.XVII.

Es un diccionario compuesto por 1.200 frases numeradas, traducidas del latín a los otros tres idiomas citados, y muy útil, como se dice en el Proemio:

Porque esto disminuira el pesado trabajo de rebolver el Vocabulario tantas vezes: esto dara a los Gramaticos y Rhetoricos mas abundante copia de palabras en tres meses solos, que otra cualquier cosa en todos tres años.

Estudiando las fuentes de la Nomenclatura he colocado a Howell dentro de esa amplia tradición de diccionarios glosados, de los pocos que recopilaron autores ingleses, ya que si exceptuamos a nuestro autor y a Minsheu no sabemos de ninguno que lo haya hecho directamente. Y tomando los dos diccionarios, a pesar de todas las reservas con las que los he juzgado, hemos de admitir que llenan el vacío lexicográfico de gran parte del siglo XVII. Hay que esperar a los primeros años del siglo siguiente para que aparezca un nuevo diccionario que no era sino una copia de los de Minsheu y Oudin (edición de 1645). Su autor fue el Capitán John Stevens que lo publicó en 1705, y constaba de 103 páginas y unas 20.000 entradas. En 1706 publica otro, mucho más ambicioso y monumental, que incluye un diccionario Inglés-Español y unos 2.000 refranes.

\section{REFERENCIAS BIBLIOGRÁFICAS}

Alonso, A. (1951). "Reseñas. Samuel Gili Gaya: Tesoro lexicográfico, 1492-1726. Madrid, CSIC, 1947 (y 1948)”. Nueva Revista de Filología Hispánica 5, 324-328.

Boulard, C. B. (1933). "The Spanish Schoole-Master and the polyglot derivatives of Noël de Berlaimont's Vocabulare". Revue Hispanique 81, 283-318.

Howell, J. (1662). A New English Grammar... There is also another Grammar of the Spanish or Castilian Toung... Whereunto is annexed A Discours or Dialog containing a Perambulation of Spain and Portugall, Londres, Printed for T. Williams, H. Brome and H. Marsh.

MARtín-GAmero, S. (1961). La enseñanza del inglés en España (desde la Edad Media hasta el siglo XIX), Madrid, Gredos.

Mumby, A. F. (1974). Publishing and Bookselling: from the Earliest Times to 1870, Londres, J. Cape.

PlOMER, H. R. (1907). A Dictionary of the Booksellers and Printers who were at work in England, Scotland and Ireland from 1641 to 1667, Londres. 
SÁNCHEZ EsCRIBANO, F. J. (1979). James Howell: un hispanista inglés del siglo XVII. Tesis doctoral sin publicar, Universidad de Zaragoza,

SÁNCHEZ EsCRIBANO, F. J. (1983). "Los diccionarios políglotos de James Howell: fuentes y aportaciones personales". Atlantis 5, 5-22.

SteINER, R. J. (1970). Two Centuries of Spanish and English Bilingual Lexicography, 1590-1800, La Haya / París, Mouton.

VerdeYen, R. (1925-1935). "Colloquia et Dictionariolum Septem Linguarum. Gedrukt door fickaert te Antwerpen in 1616 opnieuwuitgegeven door Prof. Dr. R. Verdeyen". Uitgave van de Vereeniging der Antwerpsche Bibliophilen 39 (I, 1926), 40 (II, 1925) y 42 (III, 1935).

WIENER, L. (1899). "Spanish Studies in England in the Sixteenth and Seventeenth Centuries". The Modern Quarterly of Language and Literature 2/5, 3-10.

Francisco Javier Sánchez Escribano fsanchez@unizar.es

Dpto. de Filología Inglesa y Alemana

Facultad de Filosofía y Letras

50009 Zaragoza 